$\underline{\underline{-}}$ WILHELMS-UNIVERSITÄT

\section{Strain-tuning of Dirac states at the SnTe (001) surface}

M. Drüppel, P. Krüger, M. Rohlfing

Institut für Festkörpertheorie, University of Münster, Germany

\title{
Introduction
}

- Topological insulator ( $\mathrm{TI})$ [1]: Time reversal symmetry ensures gapless surface states

- Topological crystalline insulator $(\mathrm{TCl})[2,3]$ : Mirror symmetry protects gapless surface states - New opportunity: manipulating Dirac states by breaking mirror symmetry

- SnTe first system to be identified as a TCl [2]

- Goal: Investigate stability of surface states under lattice deformations that break underlying mirror symmetries in the $\mathrm{TCl} \mathrm{SnTe}$

\section{Methods}

- GGA-DFT (PW91) with basis set of localized Gaussian orbitals

- Spin-orbit coupling included in pseudopotentials

- Slab calculations with up to 51 layers and $20 \AA$ vacuum, using the experimental lattice constant $a_{\text {exp }}=6.327 \AA$

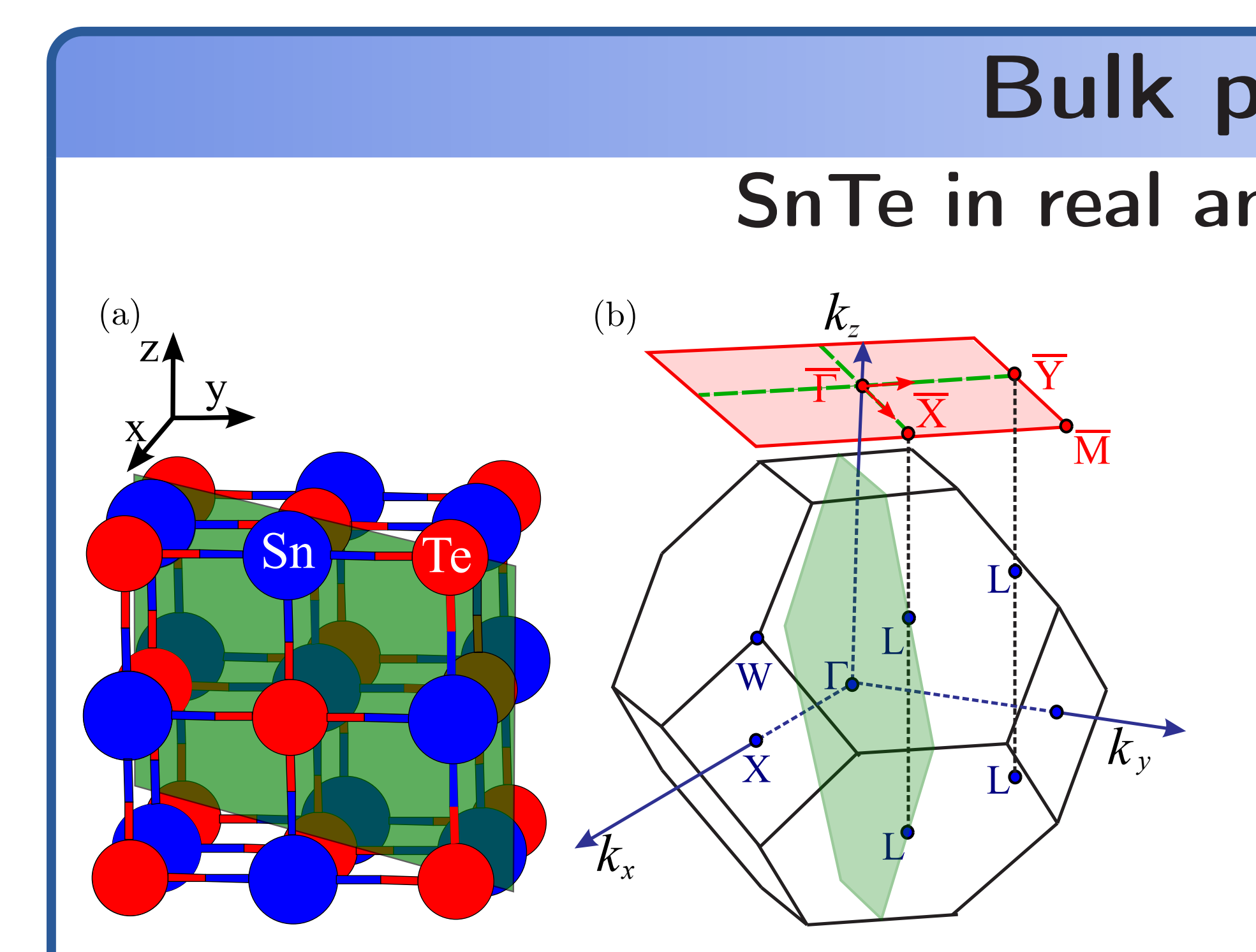

\section{Bulk properties}

\section{Bulk band structure}
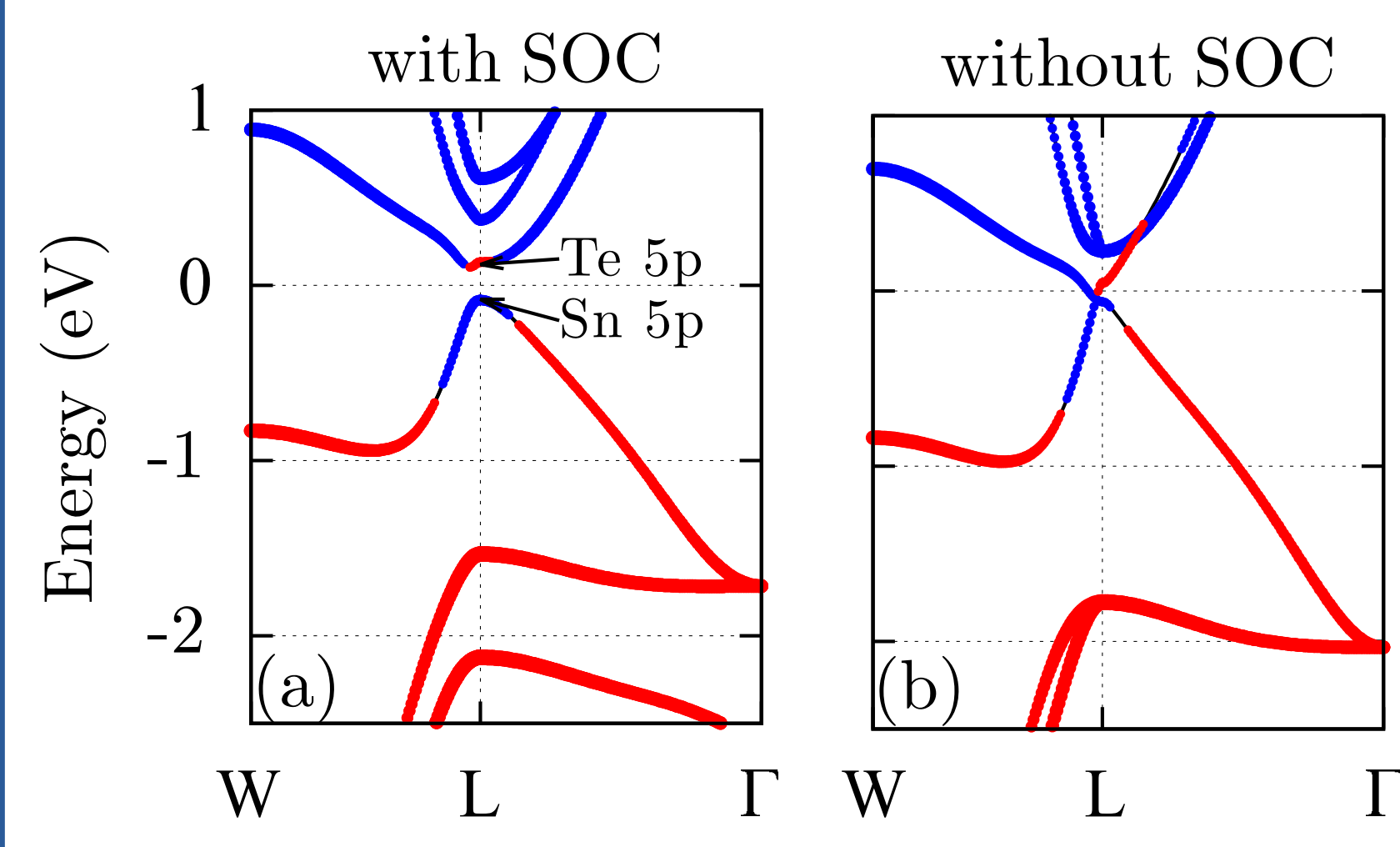

- Band structure with localization on Sn and Te atoms shows inversion at L-point (a) $\rightarrow$ Origin of $\mathrm{TCl}$ phase

- Inversion is not driven by Spin-OrbitCoupling (SOC) (b)

- But without SOC, gap remains closed

\section{(001) surface states}

Surface band structure

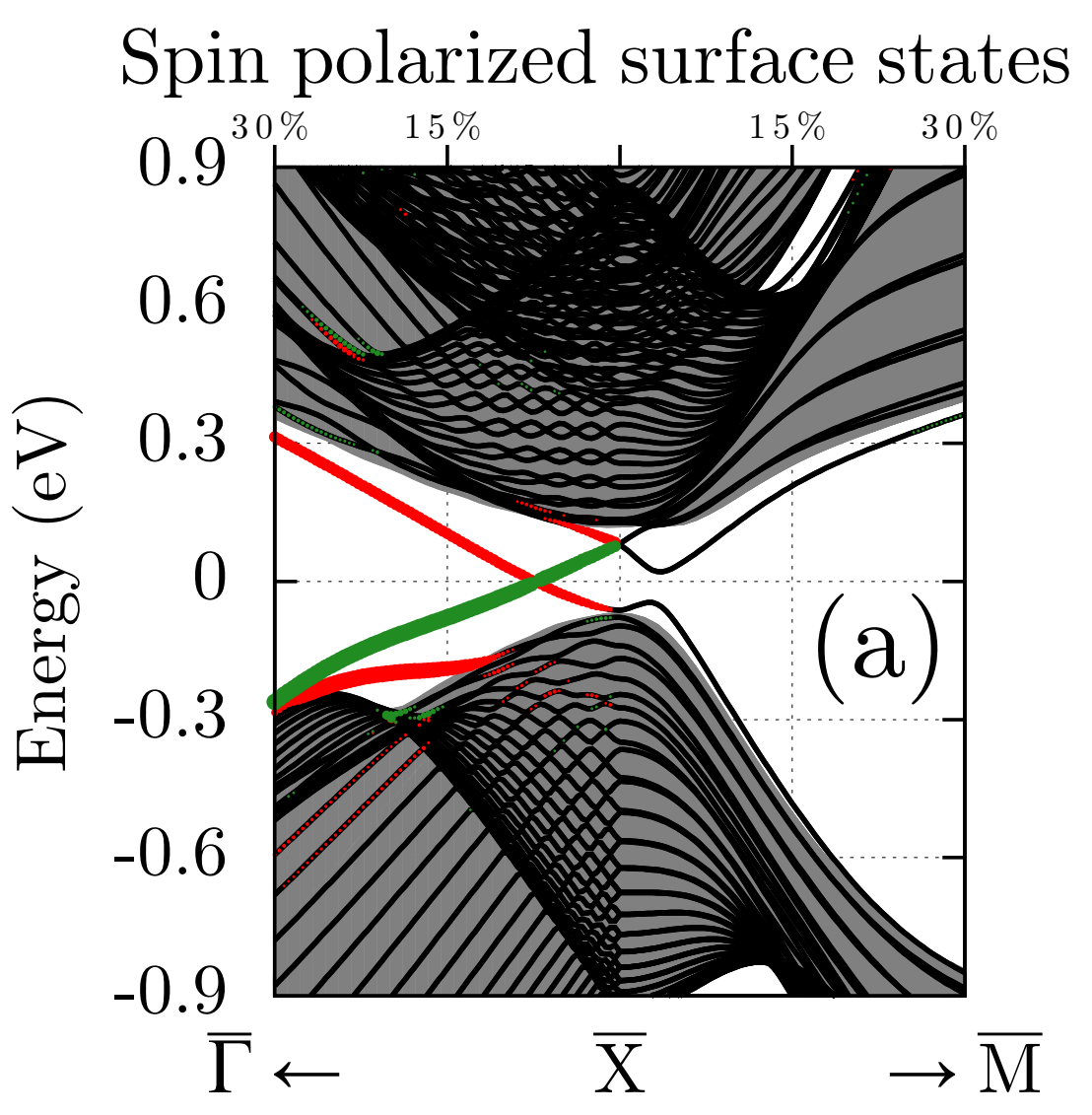

- (001) surface exhibits four spin polarized Dirac cones close to $\bar{X}$ and $\bar{Y}$ with positive/negative Rashba component of spin polarization

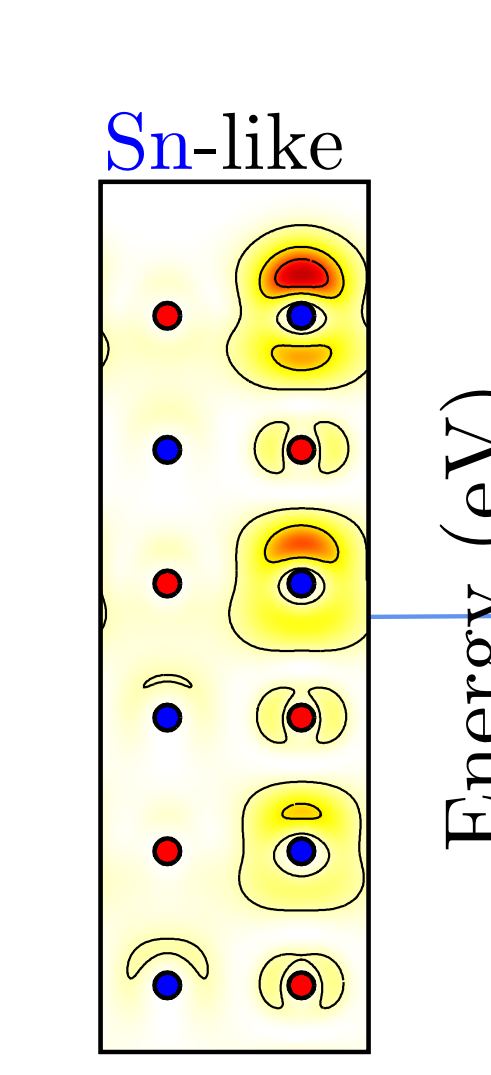

$0.9^{30}$
0.6
0.3
0
-0.3
-0.6
-0.9
$\bar{\Gamma}$

.

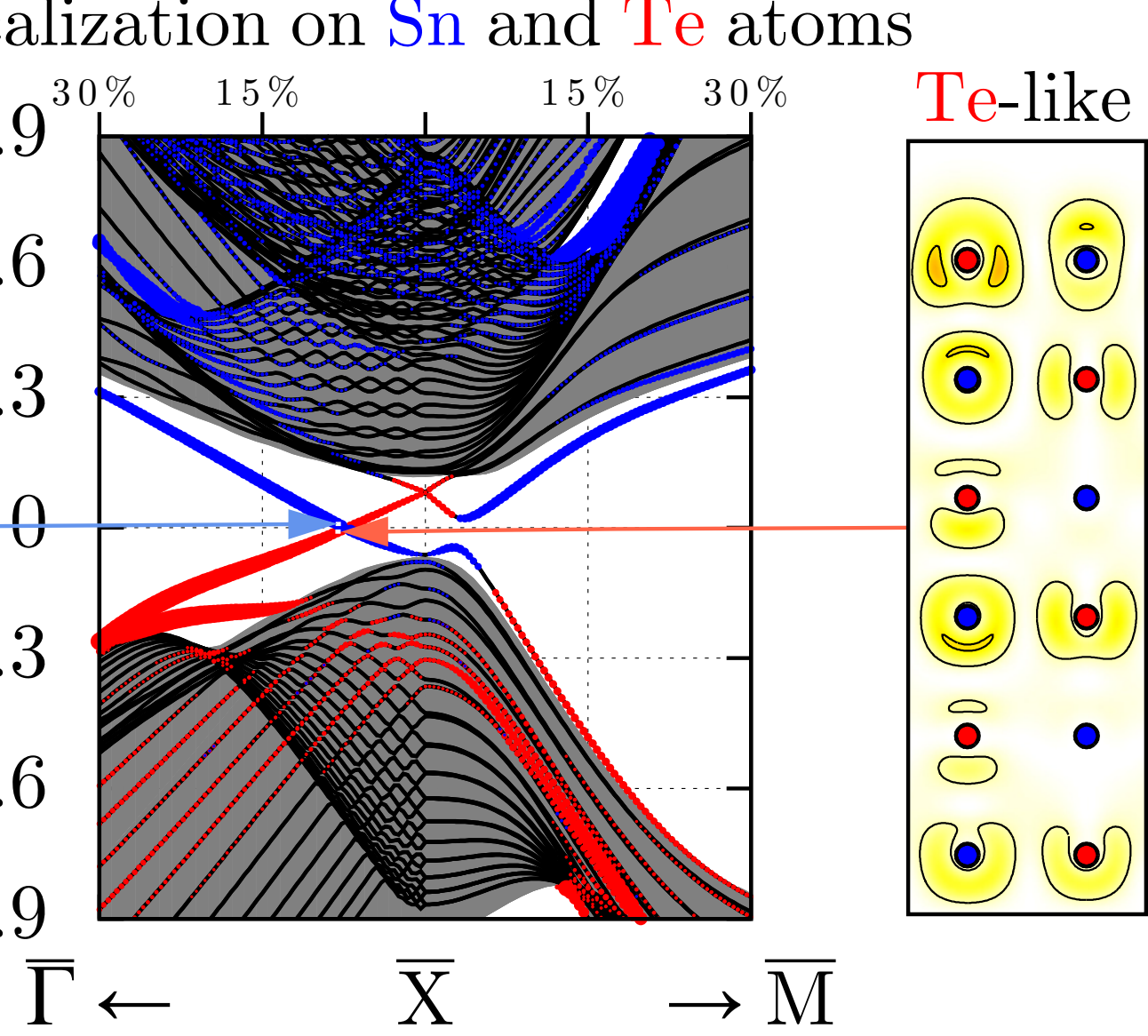

- Sn-like band, mainly Sn $5 \mathrm{p}_{z}$ orbitals

- Te-like band, mainly Te $5 p_{x}$ and $\mathrm{Sn} 5 \mathrm{~s}$ orbitals

- $\bar{\Gamma} \bar{X}$ (projection of mirror plane): bands cross and form Dirac cone

- $\overline{\mathrm{X}} \overline{\mathrm{M}}$ : coupling $\rightarrow$ anti-crossing and gap opening

Finite-size effects in SnTe films

- Overlap of states on both slab surfaces leads to hybridization and gap opening at Dirac points for finite slabs (a)

- Hybridization gap decreases exponentially with number of layers (b)

- Two different decay behaviors since surfaces are the same (different) for odd (even) number of layers
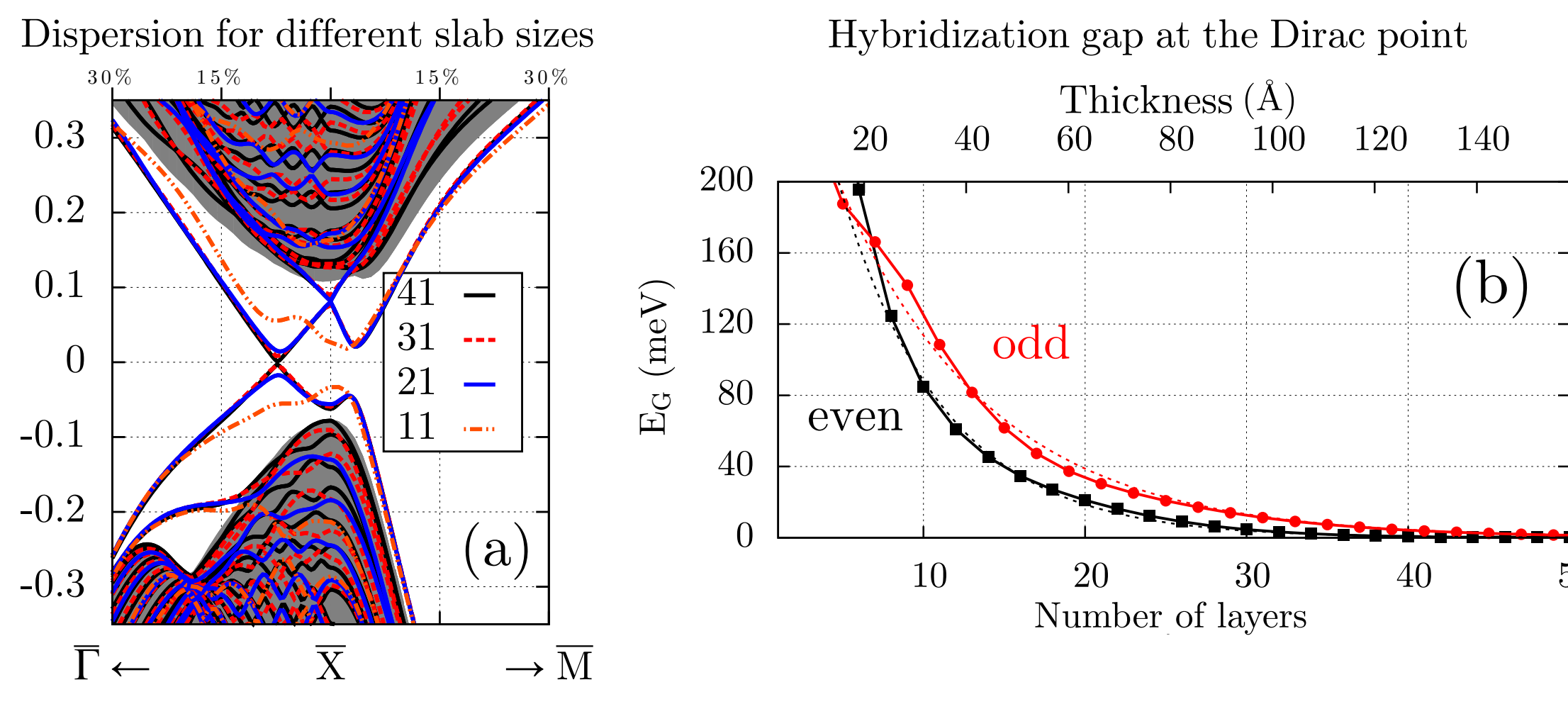

\section{Comparison with experiment}

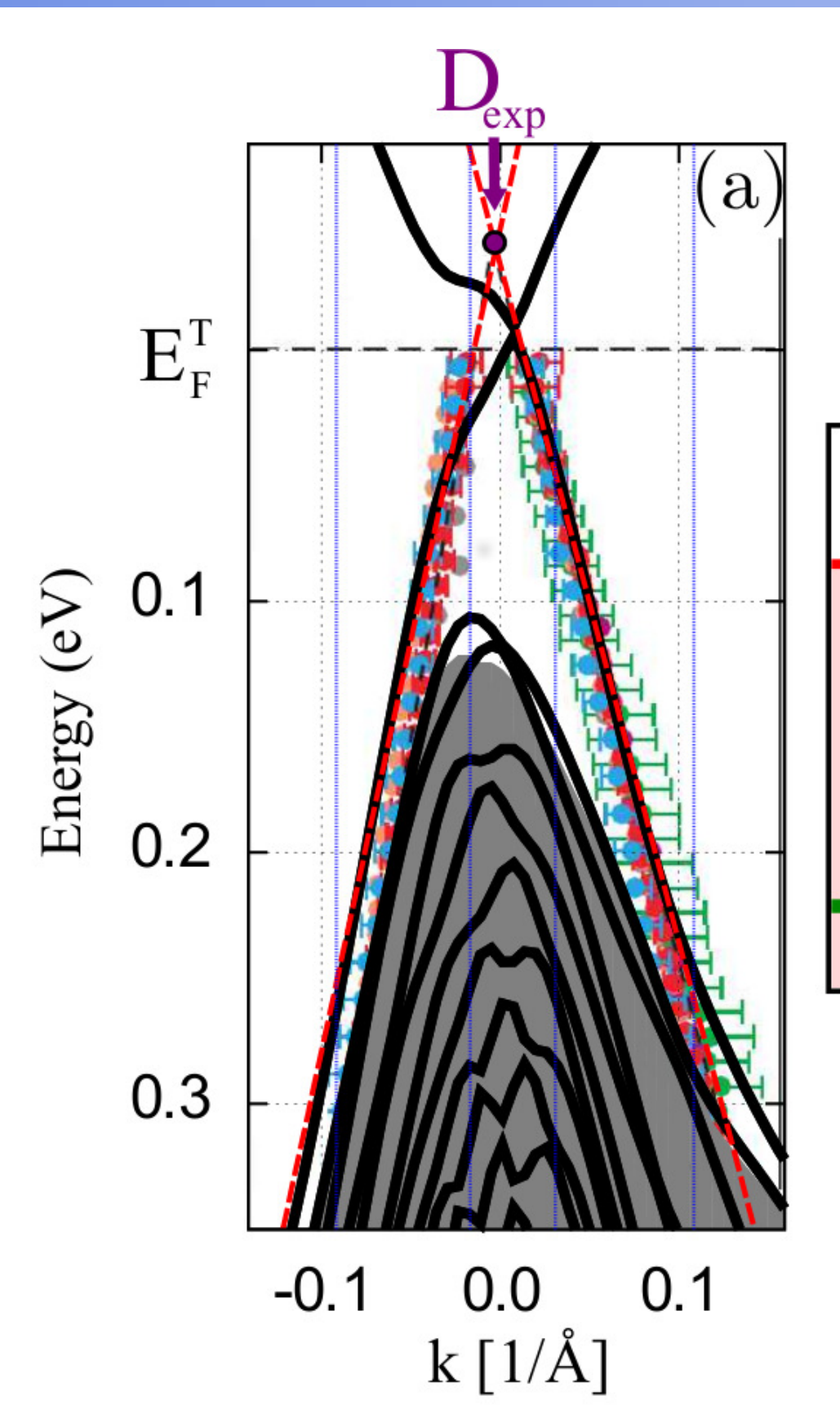

- (a) Comparison of our DFT-GGA calculations with ARPES measurements at different photon energies from Tanaka et al. [4]

- Theoretical Dirac cone $D_{\text {the }}$ lies $0.13 \frac{\pi}{a^{\prime}}$ $0.09 \AA^{-1}$ closer to $\bar{X}$ than $D_{\text {exp }}$

- (b) ARPES measurements performed along green solid arrow, calculations along green dashed arrow Exp. Dirac velocities (in units of $1 / \hbar$ ): $4.5 \mathrm{eV} \cdot \AA$ and $-3.0 \mathrm{eV} \cdot \AA$

- We find: $3.3 \mathrm{eV}$. A and $-2.7 \mathrm{eV}$. \&

- Overall good qualitative agreement

\section{Breaking mirror symmetry}

- Idea: Apply strain to SnTe for example by piezoelectric elements as suggested in Ref. [5] $\rightarrow$ Continuously deform crystal and break (110) and/or (110) mirror plane

$\rightarrow$ Induce gap at Dirac points

\section{Deformation at surface only}

- How do we break mirror symmetry? Topmost layers $(3,5$ or 8$)$ are displaced by a vector $\boldsymbol{d}$ relative to the one below (a)

- Displacement in $x^{\prime}=[110]$ direction leaves (110) plane intact (projected onto $\bar{X} \bar{\Gamma} \bar{X}$ ), but destroys (110) mirror symmetry (projected on $\bar{Y} \bar{\Gamma} \bar{Y}$ )

- Turning displacement vector by 45 degrees towards $y^{\prime}$ destroys both mirror planes

\section{(a)}
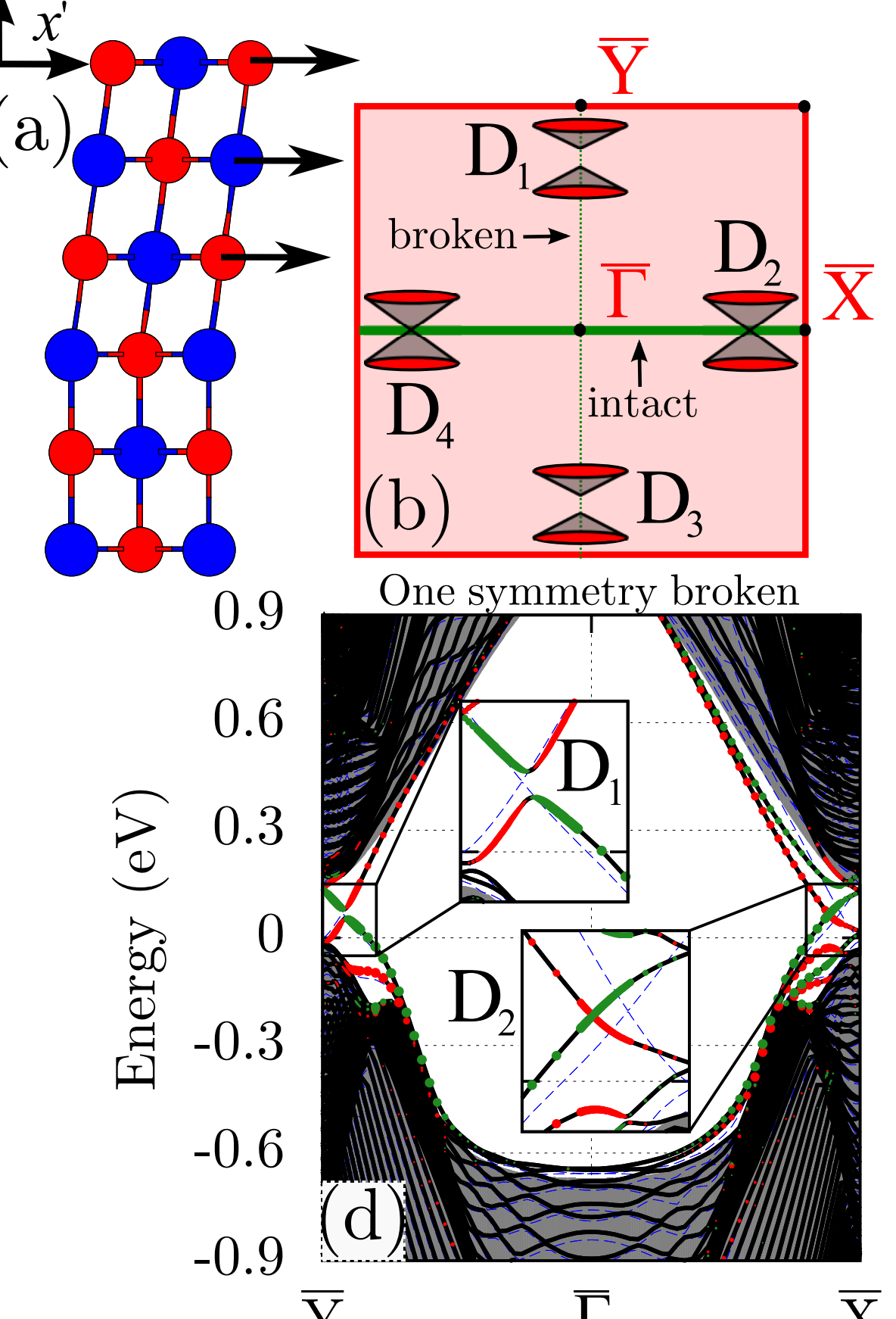

- One broken symmetry: $D_{1,3}$ become gapped $(26 \mathrm{meV})$, other two remain closed and are only slightly shifted as seen in (d) with corresponding surface BZ in (b) (first 8 layers displaced in $x^{\prime}$ direction by $0.224 \AA$ each, dashed blue lines: pristine system)

- Breaking both symmetries: All four Dirac cones are gapped by $23 \mathrm{meV}$ ((e) with BZ in (c))

Breaking mirror symmetry at the surface only can already open the gap!

Quantitative analysis of induced gap

- Magnitude of induced gap at $\mathrm{D}_{1,3}$ for only one broken mirror symmetry is shown in (a) for a displacement of 3, 5, 8 and all layers

- Effect on the surface states is too small if only three layers are displaced, which only contain $\approx 40 \%$ of charge density, no gap is opened

- In all other cases gap increases linearly for small deformations

- Maximum is reached before non-linear distortions to the electronic structure lead to decrease of the gap

- Similar situation is found if both mirror symmetries are broken (b)

- Deformation of the complete slab (blue graph): gap opens only slightly, then closes completely for all deformations

$\rightarrow$ Something more drastic happens to the electronic
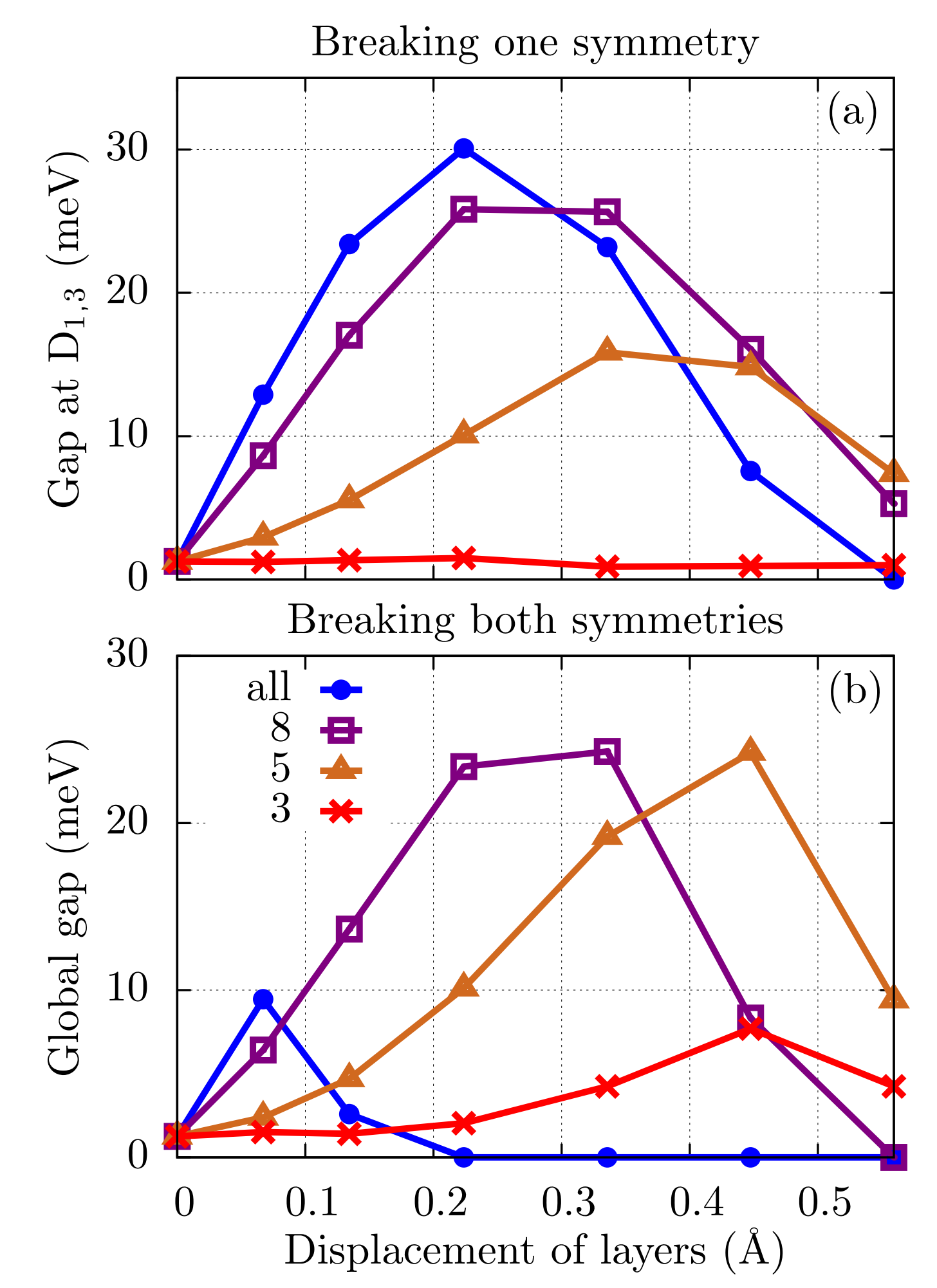
structure

Deformation of the complete slab
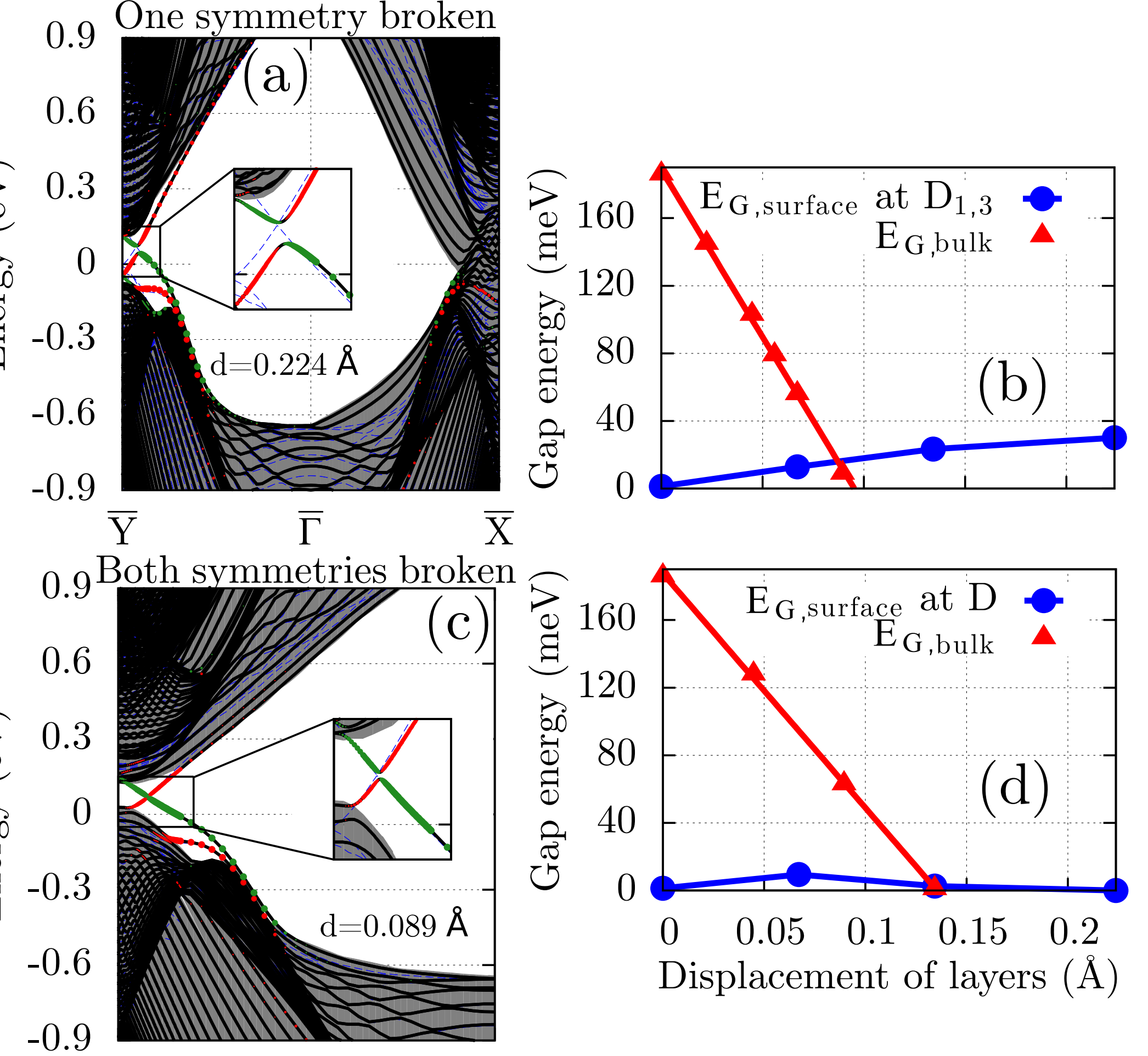

- Displacement of layers as before but now for complete slab

- Breaking one symmetry: Gap is induced at Dirac cones $\mathrm{D}_{1,3}$, see inset (a), magnitude blue graph in (b)

- At the same time bulk bands are pushed into the gap around $\bar{X}$ making the whole system metallic red graph (b)

- Breaking both symmetries: Gap opens slightly (c) at all Dirac cones, then gets closed by the bulk bands which push into the gap, (d) blue graph

\section{Conclusion}

- Breaking mirror symmetry in the TCI SnTe at the surface only can already open the gap

- Displacement of layers leads to linearly tunable gap of up to $\approx 30 \mathrm{meV}$ at the Dirac-points

- Direction of displacement controls if only two or all four Dirac cones become gapped

- Deformation of whole slab: bulk bands are pushed into the bulk gap, making the complete system metallic $\rightarrow$ Transport would be dominated by bulk carriers!

For more Details see Phy. Rev. B 90, 155312

\section{References}

[1] M. Z. Hasan and C. L. Kane. Rev. Mod. Phys. 82 (4 2010), pp. 3045-3067.

[2] T. H. Hsieh et al. Nat. Commun. 3 (2012), p. 982.

[3] L. Fu. Phys. Rev. Lett. 106 (10 2011), p. 106802.

[4] Y. Tanaka et al. Nat. Phys. 8 (2012), pp. 800-803. 